\title{
Dynamic beam splitter employing an all-dielectric metasurface based on an elastic substrate
}

 \\ ${ }^{1}$ NANOTAM-Nanotechnology Research Center, Bilkent University, 06800 Ankara, Turkey \\ ${ }^{2}$ Department of Electrical and Electronics Engineering, and Department of Physics, Bilkent University, 06800 Ankara, Turkey \\ ${ }^{3}$ UNAM-Institute of Materials Science and Nanotechnology, Bilkent University, 06800 Ankara, Turkey \\ ${ }^{4}$ Department of Electrical and Electronics Engineering, TOBB University of Economics and Technology, Ankara 06560, Turkey \\ ${ }^{5}$ Nanophotonics Research Laboratory, TOBB University of Economics and Technology, Ankara 06560, Turkey \\ ${ }^{6}$ e-mail: drhasankocer@gmail.com \\ *Corresponding author: hasan.kocer@bilkent.edu.tr
}

Received 16 March 2020; revised 12 May 2020; accepted 21 May 2020; posted 27 May 2020 (Doc. ID 392872$) ;$ published 25 June 2020

\begin{abstract}
Beam splitters are an indispensable part of optical measurements and applications. We propose a dynamic beam splitter incorporating all-dielectric metasurface in an elastic substrate under external mechanical stimulus of stretching. The optical behavior at $720 \mathrm{~nm}$ wavelength shows that it can be changed from a pure optical-diode-like behavior to a dynamic beam splitter. Although the structure is designed running at $720 \mathrm{~nm}$, the design approach with appropriate materials can be used at any wavelength. Various cases, including wavelength and polarization dependencies, are thoroughly investigated to demonstrate the principles of operating conditions of two different regimes of the designed metasurface. () 2020 Optical Society of America
\end{abstract}

https://doi.org/10.1364/OL.392872

A beam splitter has numerous uses in photonics to decompose or recompose various properties of incoming light, such as frequency, intensity, and polarization. Therefore, it is an important part of many optical experimental and measurement systems, such as fiber optics and interferometers [1]. Beam splitters can be developed using a variety of methods and structures like gratings [2,3], photonic crystals [4-7], wave plates [8,9], and metasurfaces $[1,10-12]$. Bulky and heavy beam splitters made from conventional optical components (i.e., prisms and flat glass plates) prevent their planar integration into miniature optical circuits. Recent developments in the two- dimensional subwavelength structures (metasurfaces) bring about solutions to these problems. Many experimental metasurfaces were demonstrated in the infrared (IR), visible, and even ultraviolet wavelengths [13-16]. The metasurfaces can be designed as periodic structures which are generally utilized as spectral filters, perfect absorbers, and polarization converters [17-20]. On the other hand, the gradient metasurfaces are useful for beam splitting, flat lenses, electromagnetic virtual shaping and computer-generated holography, etc. [21-26]. More recently, there has been growing interest in active metasurfaces where optical properties can be controlled dynamically after fabrication $[27,28]$ by the application of external stimuli, such as electrical [29,30], thermal [31,32], optical [33], or mechanical $[34,35]$.

In this Letter, we propose a dynamic beam splitter incorporating an all-dielectric metasurface in an elastic substrate. The beam splitter is designed to operate at $\lambda=720 \mathrm{~nm}$, but the design approach can be utilized at any wavelength provided that suitable materials are available. By means of an external mechanical stimulus applied to the elastic substrate, the behavior of the proposed structure can be changed from a pure optical-diode-like behavior (where the transmission is blocked) to a dynamic beam splitter in which the incoming light power is split three ways with different split angles and power ratios.

To implement the proposed structure, we utilized a phase gradient all-dielectric metasurface composed of periodically arranged binary unit cells where the difference of the phase delay is $\pi$ radian [1]. The corresponding unit cell is shown in Fig. 1(a). The unit cell consists of a circular cross-sectional nanopillar that is made up of high-index silicon ( $\mathrm{Si}$ ) on a thin layer of aluminum oxide $\left(\mathrm{Al}_{2} \mathrm{O}_{3}\right)$ in an elastic and low-index polydimethylsiloxane (PDMS) substrate. In order to fulfill $2 \pi$ phase coverage, the heights of the $\mathrm{Si}$ and $\mathrm{Al}_{2} \mathrm{O}_{3}$ are fixed as $h_{\mathrm{Si}}=500 \mathrm{~nm}$ and $h_{\mathrm{Al} 2 \mathrm{O} 3}=100 \mathrm{~nm}$, respectively. Then the radius of the nanopillar $(r)$, and unit cell period $(P)$ are varied in the numerical electromagnetic simulations utilizing Lumerical, a commercially available finite-difference time-domain (FDTD) simulation software package. The monochromatic light source $\left(\lambda_{i}=720 \mathrm{~nm}\right.$ red light) is applied from the backward direction (across PDMS to the nanopillar). It is a linearly polarized plane wave along the $x$ direction and normally incident to the unit cell. The spectral refractive indices of $\mathrm{Si}$ and $\mathrm{Al}_{2} \mathrm{O}_{3}$ were taken from the Palik database. Since the PDMS is a flexible and lossless substrate in the wavelength of interest, its refractive index is assigned as a constant value of 1.41 [35] in all simulations throughout this Letter. Perfectly matched layers along the $z$ axis and periodic boundary conditions along the $x$ and $y$ axes are chosen in the simulations. $P$ is changed from 300 to $500 \mathrm{~nm}$ with $\Delta P=5 \mathrm{~nm}$ steps, while the $r$ of the nanopillar is swept from 50 to $125 \mathrm{~nm}$ with $\Delta r=1 \mathrm{~nm}$ steps. The resulting map for transmitted and incident power ratio is 




Fig. 1. (a) Schematic drawing of the $3 \mathrm{D}$ unit cell. (b) Transmission and (c) phase maps according to varying $P$ and $r$ at $\lambda_{i}=720 \mathrm{~nm}$. (d) Transmission and phase characteristics at $P=300 \mathrm{~nm}$ and $\lambda_{i}=720 \mathrm{~nm}$ with respect to $r$. The inset shows the top view of the designed unit cell.

given as the "transmission" in Fig. 1(b). Likewise, the phase of the transmitted electric field is computed as the "phase" map in Fig. 1(c). By using the phase and the transmission maps, we select the optimum value of $P=300 \mathrm{~nm}$ along the $x$ and $y$ directions to get high transmission and $2 \pi$ phase coverage. Then we obtain the radius dependence of the transmission and phase values in Fig. 1(d).

After designing the unit cell, we construct a phase gradient metasurface along the $x$ axis in the top layer for dynamic beam splitter operation as illustrated in Fig. 2(a).

The top layer consists of the periodic arrangement of a supercell enclosed in dashed black lines where two nanopillars of $r_{1}$ and $r_{2}$ radii $\left(r_{2}>r_{1}\right)$ exist. The phase difference between two successive radii along the $x$ axis $(\Delta \Phi(x))$ is selected at a fixed value of $\pi$ radian [1]. Based on this requirement, we pick $r_{1}=60 \mathrm{~nm}$ and $r_{2}=83 \mathrm{~nm}$ from Fig. 1(d). This radii pair

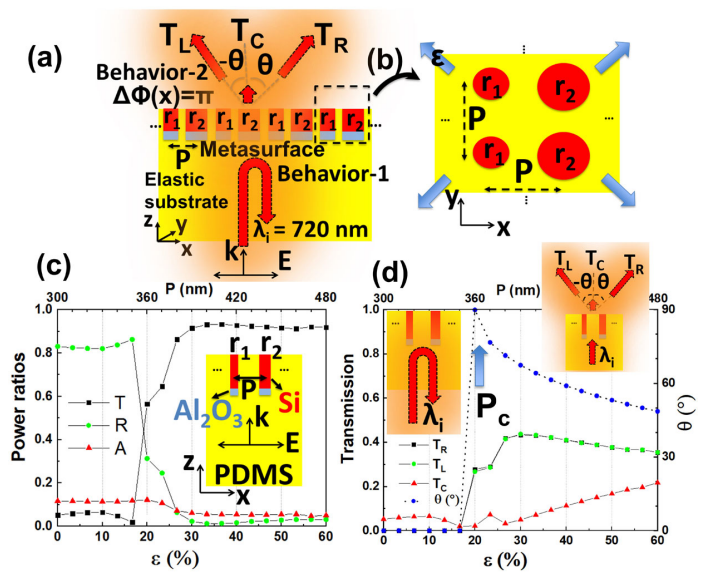

Fig. 2. (a) $x-z$ side view of the proposed dynamic beam splitter. (b) $x-y$ top view of the supercell under diagonal stretching with a strain ratio of $\varepsilon$. (c) Transmission $(T)$, reflection $(R)$, and absorption $(A)$ power ratios of the design with respect to varying period $(P)$ under different strain levels $(\varepsilon)$ at $\lambda_{i}=720 \mathrm{~nm}$. The inset shows the $x-z$ side view of the supercell. (d) Right/left split $\left(T_{R} / T_{L}\right)$, and non-split $\left(T_{C}\right)$ power ratios and splitting angles $(\theta)$ with respect to $P$ and $\varepsilon$. provides not only the constant phase difference of $\pi$ radian, but also high transmission. Note that the heights of the components of the nanopillar are taken at the same values $\left(h_{\mathrm{Si}}=500 \mathrm{~nm}\right.$, $\left.h_{\mathrm{Al} 2 \mathrm{O} 3}=100 \mathrm{~nm}\right)$ as in the unit cell design. A linearly polarized plane wave of monochromatic light $\left(\lambda_{i}=720 \mathrm{~nm}\right)$ along the $x$ axis is sent from the backward direction. Our design is targeted to have two distinct behaviors by stretching the period. Depending on the stretch level, initially observed nontransmitting or optical-diode-like behavior (Behavior-1) turns to dynamic beam splitter behavior (Behavior-2). In Behavior-2 mode, the incoming light power is dynamically divided into three distinct directions with angles $-\theta, 0, \theta$ and power ratios of left directed $\left(T_{L}\right)$, center directed $\left(T_{C}\right)$, and right directed $\left(T_{R}\right)$. As seen in the $x-y$ top view of the supercell [Fig. 2(b)], we apply a uniform diagonal stretch ratio $(1+\varepsilon)$ with a strain ratio $(\varepsilon)$ to the elastic substrate as seen in the blue arrows. We assume that the unstretched period $\left(P_{u}=300 \mathrm{~nm}\right)$, which is initially strain-free, is affected by the diagonal stretching at the same rate along the $x$ and $y$ axes. Then the period under diagonal stretching $(P)$ is described as $P=P_{u}(1+\varepsilon)$. The operation of our design under different strain values can be analytically modeled utilizing the generalized Snell's law [13] for refraction as follows:

$$
n_{t} \sin \theta_{t}-n_{i} \sin \theta_{i}=\frac{\lambda_{0}}{2 \pi} \frac{d \emptyset}{d x},
$$

where $n_{i}\left(n_{t}\right)$ is the refractive index of the incident (refracted) medium, $\theta_{i}\left(\theta_{t}\right)$ is the incident (refracted) angle, $\lambda_{0}$ is the free-space wavelength of the light, $d \Phi(x)$ is the phase difference between successive unit cells, and $d x$ is the period of the supercell. Our parameters of design at $\lambda_{i}=\lambda_{0}=720 \mathrm{~nm}$ are $n_{i}=1.41$ (PDMS), $n_{t}=1.00$ (air), $\theta_{i}=0$ (normal incidence), $d \Phi(x)=\Delta \Phi(x)= \pm \pi$ radian, $d x=P$ (the period of the supercell), and $P=P_{u}(1+\varepsilon)=300(\mathrm{~nm})(1+\varepsilon)$. Importing these parameters into Eq. (1), we can analytically model the split angles depending on the strain ratio given below:

$$
\theta=\sin ^{-1}\left(\frac{720 \mathrm{~nm}}{2 \pi * 1.00} \frac{ \pm \pi}{300 \mathrm{~nm} *(1+\varepsilon)}\right) .
$$

Starting from the condition of the unstretched period of $300 \mathrm{~nm}(\varepsilon=0)$ up to $\varepsilon=20 \%\left(P=P_{c}=360 \mathrm{~nm}\right)$, the real values of split angles cannot be obtained in Eq. (2), since the input value (inside the brackets) in the inverse sine function in Eq. (2) is larger than "1." Therefore, the incoming light cannot transmit from the substrate to the air, but it is reflected from the top metasurface layer back to the substrate. This condition of the structure is called Behavior-1. After exceeding the critical period $\left(P \geq P_{c}\right)$ by increasing the strain ratio $(\varepsilon \geq 20 \%)$, Eq. (2) is able to produce real values for the split angles. In other words, the incoming light now transmits to the air side with negligible reflection. The transmission characteristics are like a three-way beam splitter as depicted in Fig. 2(a). This condition converts the structure to Behavior-2. In Figs. 2(c) and 2(d), we numerically obtain power ratios and splitting angles with respect to varying parameters (i.e., $P$ and $\varepsilon$ ). In Fig. 2(c), we excite the structure from the elastic substrate to the top metasurface layer at $\lambda_{i}=720 \mathrm{~nm}$ with TM polarization as seen in the inset of Fig. 2(c). According to the power conservation rule, $R+T+A=1$ where $R, T$, and $A$ are power ratios for reflection, transmission, and absorption, respectively. $R$ and $T$ values are computed numerically in our design parameters 
under varying $\varepsilon$ and $P$ at $\lambda_{i}=720 \mathrm{~nm}$; then $A$ is calculated by $A=1-R-T$. Looking at these parameters, we observe that our design operates in two different modes as mentioned earlier. The absorption shows little variation during stretching as the pair $R$ and $T$ is varying opposite each other. This situation can be better understood by examining the components of transmitted power ratios in Fig. 2(d), where the transmitted power is divided into three directions with angles. When $P<P_{c}$ $(\varepsilon<20 \%)$, the design shows reflecting behavior as illustrated in the left inset of Fig. 2(d). Here a small amount of $T$ exists and equals to $T_{C}$ (less than 0.1 ). After exceeding the critical strain parameters $\left(P \geq P_{c}, \varepsilon \geq 20 \%\right)$, a three-way dynamic beam splitter operation [exhibited in the right inset of Fig. 2(d)] appears so that power ratios of $T_{R}$ and $T_{L}$ are equal to each other, but they are greater than $T_{C}$. Moreover, $T_{R}$ and $T_{L}$ are gradually decreasing in contrast to a gradual increase in $T_{C}$ after $\varepsilon=30 \%$, since the total transmission is constant in these stretched regions of interest. Figure 2(d) also depicts the numerically found splitting angles $(\theta)$ with respect to $P$ and $\varepsilon$. Meanwhile, we calculate $\theta$ analytically by using Eq. (2) and see that it gives the same result as the numerical simulation. We notice that $\theta$ is zero up to $P=P_{c}(\varepsilon=20 \%)$. Just beyond this critical point, $\theta$ jumps to 90 degrees; then it drops with respect to $\varepsilon$. This means that split powers in right and left directions have larger split angles in the onset of the Behavior-2 mode. Depending on the rise in the strain level, the split angles become narrower.

Figure 3 shows the wavelength dependency. Here we select seven different wavelengths (six in the visible and one in the near-IR region) and excite them sequentially as the backward illumination of TM polarized light [pictured in the inset of Fig. 3(d)].

It is found from Figs. 3(a) and 3(b) that our design exhibits both behavioral modes (i.e., Behavior-1 \& 2) only at the red $(720 \mathrm{~nm})$ and near-IR $(850 \mathrm{~nm})$ wavelengths, while it always stays in Behavior- 2 mode at the rest of the simulated wavelengths. This result stems from the generalized Snell's law for refraction that was explained previously. For 720 and $850 \mathrm{~nm}$ wavelengths, our design has both behaviors according to $\varepsilon$. However, the conversion conditions from Behavior-1 to Behavior-2 differ for these wavelengths as clearly seen in Figs. 3(a) and 3(b). In the red color, the critical strain ratio is $\varepsilon_{c}=20 \%\left(P_{c}=360 \mathrm{~nm}\right)$, which was discussed earlier. In the
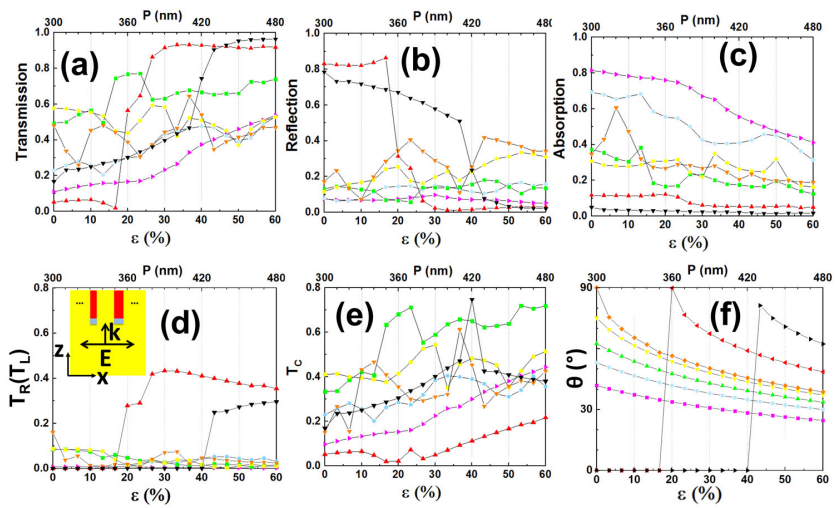

Fig. 3. (a) Transmission, (b) reflection, (c) absorption, (d) $T_{R}\left(T_{L}\right)$, (e) $T_{C}$, and (f) $\theta$ for varying $P$ and $\varepsilon$ under different wavelengths (black, $850 \mathrm{~nm}$; red, $720 \mathrm{~nm}$; orange, $600 \mathrm{~nm}$; yellow, $580 \mathrm{~nm}$; green, $532 \mathrm{~nm}$; blue, $480 \mathrm{~nm}$; violet, $400 \mathrm{~nm}$ ) of illumination.
near-IR, $\varepsilon_{c}=41.7 \%\left(P_{c}=425 \mathrm{~nm}\right)$. Since we design our structure at the red color, its properties at the red color are superior to the near-IR. Namely, Behavior-1 of the red color has less transmission and higher reflection than the near-IR. Moreover, the steepness of the behavioral transition is better in the design wavelength of the red color. In addition, the absorption plot in Fig. 3(c) shows that the longer wavelengths (red and near-IR) have negligibly small and strain-insensitive absorption, whereas the smaller wavelengths (violet and blue) have large absorptions that are inversely proportional to the strain ratio. The other colors have a moderate level of absorptions that are affected by the strain, but not as much as the violet and blue colors. These absorption contrasts for different colors and different strains originate from the highly localized electric fields inside the nanopillar in which the absorbed power loss of the main constituent element $(\mathrm{Si})$ increases as the wavelength is blueshifted, since $\mathrm{Si}$ becomes more lossy at shorter wavelengths compared to the other wavelengths of interest. Right/left split power ratios in Fig. 3(d) show that all the colors except red and near-IR are in Behavior- 2 where three-way beam splitter operation is observed, but the total transmission is mostly concentrated in the non-split component [Fig. 3(e)]. When we look at the red and near-IR wavelengths, we see that they exhibit both behaviors as follows. In Behavior- 1 mode, $T_{C}$ of near-IR is larger than $T_{C}$ of the red, because our structure is designed at the red wavelength. In Behavior- 2 mode, both colors have equal $T_{R}$ and $T_{L}$ components that are greater than their $T_{C} s$ as well. In addition, at the red wavelength in Behavior-2, we note that $T_{R}$ and $T_{L}$ power ratios are inversely proportional to $\varepsilon$, while $T_{C}$ is directly proportional to $\varepsilon$. If we switch the wavelength to near-IR, completely opposite results occur. In Fig. 3(f), we figure out that all the colors except red and near-IR have split angles decaying with respect to $\varepsilon$, since they are already transformed into Behavior-2 before $P=300 \mathrm{~nm}$. Another important observation can be extracted from Fig. 3(f) such that the split angles at any point of $\varepsilon$ are directly proportional to the excitation wavelength. In other words, the higher the wavelength, the higher the split angle. For instance, at $\varepsilon=50 \%$, as the wavelengths span from the violet to the near-IR, $\theta$ values increment from $28^{\circ}$ to $75^{\circ}$. Moreover, the ranges of split angles for these colors can be adjusted depending on the applied strain ratio. As a result, this property leads our design to have a frequency or wavelength selective splitting characteristics.

Our simulations on the polarization dependency reveal that the polarization of the light at $\lambda_{i}=720 \mathrm{~nm}$ affects the performance to a certain extent without degrading the primary performance, since the illumination coming from the substrate and impinging on the top layer does not see perfect symmetry due to the gradient metasurface in the top layer. The effect of the polarization change is the most pronounced in the range of $\varepsilon=25 \%$ to $50 \%$ in which the structure is at Behavior-2. We also observe that our structure is nearly polarization-independent at Behavior-1 $(\varepsilon<20 \%)$.

The dynamic feature of the proposed structure is summarized visually and numerically in an example in Fig. 4. Under no strain case in Fig. 4(a), our structure exhibits a non-transmitting case of an optical-diode-like behavior at $\lambda_{1}$ and $\lambda_{2}$. This case is also supported with high values of reflections $(0.83$ and 0.78$)$ in Fig. 4(d). If the illumination direction is changed to forward direction, the transmitting case of the optical-diode-like behavior is observed (i.e., $T=0.75, R=0.19$ for $\lambda_{1}=720 \mathrm{~nm}$ ). If 


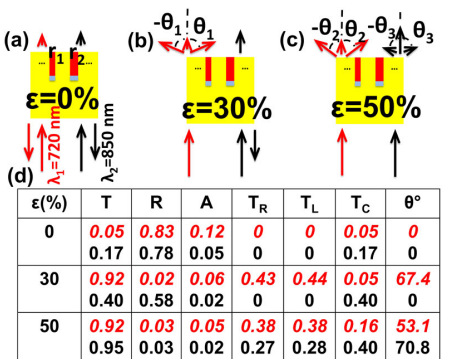

Fig. 4. Different behaviors for two different wavelengths $\left(\lambda_{1}=720 \mathrm{~nm}\right.$ and $\left.\lambda_{2}=850 \mathrm{~nm}\right)$ under (a) $\varepsilon=0 \%$, (b) $\varepsilon=30 \%$, and (c) $\varepsilon=50 \%$. (d) Quantitative values of power ratios and split angles.

we change $\varepsilon=30 \%$ as in Fig. 4(b), $\lambda_{1}$ shows three-way beam splitter behavior $\left(T_{R, L}\right.$ is higher than $40 \%$ with split angles of $67.4^{\circ}$ ), while the $\lambda_{2}$ still stays in a mostly reflecting situation. If we further raise the level of the strain to $\varepsilon=50 \%$ as illustrated in Fig. 4(c), both wavelengths of interest behave as three-way beam splitters with different split angles similar to optical spectrometer behavior in which $\lambda_{1}$ and $\lambda_{2}$ split with different angles of $53.1^{\circ}$ and $70.8^{\circ}$, respectively. With the existing nanofabrication techniques described in various experimental dynamic metasurfaces [35-37] where the experimental strain levels exerted on the PDMS and the operating wavelengths are similar to ours, there is no doubt that proposed structure is experimentally achievable.

In conclusion, we have proposed a dynamic beam splitter incorporating all-dielectric metasurface in an elastic PDMS substrate. The proposed structure was designed at a red wavelength of $720 \mathrm{~nm}$. Upon the exertion of the mechanical strain levels from $0 \%$ up to $50 \%$ on the elastic substrate, the behavior of the structure can purposely be transformed from an opticaldiode-like behavior to a three-way beam splitter in which transmission properties (right/left split and central non-split power ratios, and splitting angles) vary dynamically with respect to the applied strain level. This split mechanism is also modeled analytically. Then we made further numerical simulations to understand the wavelength and polarization dependencies. Since our structure is designed at the wavelength of $720 \mathrm{~nm}$, the properties at this wavelength are superior to other colors. However, the design approach with proper materials is applicable to any desired wavelength in the optical, and even in the microwave bands. Another aspect of the design has shown that the split angles at any strain level are directly proportional to the excitation wavelength. In other words, the higher the wavelength, the higher the split angle. As a result, this feature makes our design capable of dynamically tunable and selective separation of frequency or wavelength.

Disclosures. The authors declare no conflicts of interest.

\section{REFERENCES}

1. A. Ozer, N. Yilmaz, H. Kocer, and H. Kurt, Opt. Lett. 43, 4350 (2018).

2. Z. Wang, Y. Tang, L. Wosinski, and S. He, IEEE Photonics Technol. Lett. 22, 1568 (2010).

3. D. Taillaert, H. Chong, P. Borel, L. Frandsen, R. D. L. Rue, and R. Baets, IEEE Photonics Technol. Lett. 15, 1249 (2003).

4. S. Feng, T.-H. Xiao, L. Gan, and Y.-Q. Wang, J. Phys. D 50, 025107 (2017).
5. P. Qiu, W. Qiu, Z. Lin, H. Chen, J. Ren, J.-X. Wang, Q. Kan, and J.-Q. Pan, Sci. Rep. 7, 9588 (2017).

6. U. G. Yasa, I. H. Giden, M. Turduev, and H. Kurt, J. Opt. 19, 095005 (2017).

7. U. G. Yasa, M. Turduev, I. H. Giden, and H. Kurt, J. Lightwave Technol. 35, 1677 (2017).

8. R.-C. Tyan, A. A. Salvekar, H.-P. Chou, C.-C. Cheng, A. Scherer, P.-C. Sun, F. Xu, and Y. Fainman, J. Opt. Soc. Am. A 14, 1627 (1997).

9. F. Ding, Z. Wang, S. He, V. M. Shalaev, and A. V. Kildishev, ACS Nano 9, 4111 (2015).

10. M. Wei, Q. Xu, Q. Wang, X. Zhang, Y. Li, J. Gu, Z. Tian, X. Zhang, J. Han, and W. Zhang, Appl. Phys. Lett. 111, 071101 (2017).

11. D. Zhang, M. Ren, W. Wu, N. Gao, X. Yu, W. Cai, X. Zhang, and J. Xu, Opt. Lett. 43, 267 (2018).

12. B. Wang, F. Dong, H. Feng, D. Yang, Z. Song, L. Xu, W. Chu, Q. Gong, and Y. Li, ACS Photonics 5, 1660 (2018).

13. N. Yu, P. Genevet, M. A. Kats, F. Aieta, J.-P. Tetienne, F. Capasso, and Z. Gaburro, Science 334, 333 (2011).

14. M. Pu, X. Li, X. Ma, Y. Wang, Z. Zhao, C. Wang, C. Hu, P. Gao, C. Huang, H. Ren, X. Li, F. Qin, J. Yang, M. Gu, M. Hong, and X. Luo, Sci. Adv. 1, e1500396 (2015).

15. T. Siefke, S. Kroker, K. Pfeiffer, O. Puffky, K. Dietrich, D. Franta, I. Ohlidal, A. Szeghalmi, E.-B. Kley, and A. Tunnermann, Adv. Opt. Mater. 4, 1780 (2016).

16. S. Wang, X. Ouyang, Z. Feng, Y. Cao, M. Gu, and X. Li, Opto-Electron. Adv. 1, 170002 (2018)

17. M. Pu, P. Chen, Y. Wang, Z. Zhao, C. Huang, C. Wang, X. Ma, and X. Luo, Appl. Phys. Lett. 102, 131906 (2013).

18. N. K. Grady, J. E. Heyes, D. R. Chowdhury, Y. Zeng, M. T. Reiten, A. K. Azad, A. J. Taylor, D. A. R. Dalvit, and H.-T. Chen, Science 340, 1304 (2013).

19. X. Ma, C. Huang, M. Pu, C. Hu, Q. Feng, and X. Luo, Opt. Express 20, 16050 (2012).

20. O. Hemmatyar, S. Abdollahramezani, Y. Kiarashinejad, M. Zandehshahvar, and A. Adibi, Nanoscale 11, 21266 (2019).

21. M. Khorasaninejad, W. T. Chen, R. C. Devlin, J. Oh, A. Y. Zhu, and F. Capasso, Science 352, 1190 (2016).

22. M. Pu, Z. Zhao, Y. Wang, X. Li, X. Ma, C. Hu, C. Wang, C. Huang, and X. Luo, Sci. Rep. 5, 9822 (2015).

23. X. Li, L. Chen, Y. Li, X. Zhang, M. Pu, Z. Zhao, X. Ma, Y. Wang, M. Hong, and X. Luo, Sci. Adv. 2, e1601102 (2016).

24. X. Ni, Z. J. Wong, M. Mrejen, Y. Wang, and X. Zhang, Science 349, 1310 (2015).

25. Y. Guo, L. Yan, W. Pan, and L. Shao, Sci. Rep. 6, 30154 (2016).

26. P. Genevet, F. Capasso, F. Aieta, M. Khorasaninejad, and R. Devlin, Optica 4, 139 (2017).

27. H. Hajian, A. Ghobadi, A. E. Serebryannikov, B. Butun, G. A. E. Vandenbosch, and E. Ozbay, J. Opt. Soc. Am. B 36, 1607 (2019).

28. S. Abdollahramezani, O. Hemmatyar, H. Taghinejad, A. Krasnok, Y. Kiarashinejad, M. Zandehshahvar, A. Alu, and A. Adibi, arXiv:2001.06335v1 (2020).

29. Y. Yao, R. Shankar, M. A. Kats, Y. Song, J. Kong, M. Loncar, and F. Capasso, Nano Lett. 14, 6526 (2014).

30. P. C. Wu, R. A. Pala, G. K. Shirmanesh, W.-H. Cheng, R. Sokhoyan, M. Grajower, M. Z. Alam, D. Lee, and H. A. Atwater, Nat. Commun. 10, 1 (2019).

31. M. J. Dicken, K. Aydin, I. M. Pryce, L. A. Sweatlock, E. M. Boyd, S. Walavalkar, J. Ma, and H. A. Atwater, Opt. Express 17, 18330 (2009).

32. H. Kocer, A. Ozer, S. Butun, K. Wang, J. Wu, H. Kurt, and K. Aydin, IEEE J. Sel. Top. Quantum Electron. 25, 4700607 (2019).

33. M. R. Shcherbakov, S. Liu, V. V. Zubyuk, A. Vaskin, P. P. Vabishchevich, G. Keeler, T. Pertsch, T. V. Dolgova, I. Staude, I. Brener, and A. A. Fedyanin, Nat. Commun. 8, 17 (2017).

34. I. M. Pryce, K. Aydin, Y. A. Kelaita, R. M. Briggs, and H. A. Atwater, Nano Lett. 10, 4222 (2010).

35. S. Mahsa, K. E. Arbabi, A. Arbabi, Y. Horie, and A. Faraon, Laser Photonics Rev. 10, 1002 (2016).

36. S. C. Malek, H. S. Ee, and R. Agarwal, Nano Lett. 17, 3641 (2017).

37. P. Gutruf, C. Zou, W. Withayachumnankul, M. Bhaskaran, S. Sriram, and C. Fumeaux, ACS Nano 10, 133 (2016). 